\title{
Energy transfer processes in semiconductor quantum dots - bacteriorhodopsin hybrid system
}

\author{
Aliaksandra Rakovich*a, Yury Rakovich ${ }^{\mathrm{a}}$, Igor Nabiev ${ }^{\mathrm{b}}$ and John F. Donegan ${ }^{\mathrm{a}}$

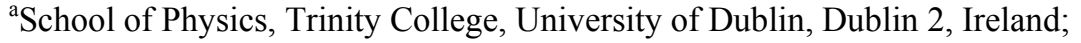 \\ ${ }^{\mathrm{b}}$ Université de Reims Champagne-Ardenne, 51 rue Cognacq Jay, 51100 Reims, France \\ *To whom correspondence should be addressed. Tel.: (++353)18964640, email: rakovica@tcd.ie
}

\begin{abstract}
The potential impact of nanoscience on energy transfer processes in biomolecules was investigated on the example of a complex between fluorescent semiconductor nanocrystals and photochromic membrane protein. The interactions between colloidal CdTe quantum dots (QDs) and bacteriorhodopsin (bR) protein were studied by a variety of spectroscopic techniques, including integrated and time-resolved fluorescence spectroscopies, zeta potential and size measurement, and fluorescence correlation spectroscopy. QDs' luminescence was found to be strongly modulated by bacteriorhodopsin, but in a controllable way. Decreasing emission lifetimes and blue shifts in QDs' emission at increasing protein concentrations suggest that quenching occurs via Förster resonance energy transfer. On the other hand, concave Stern-Volmer plots and sigmoidal photoluminescence quenching curves imply that the self-assembling of NCs and bR exists, and the number of nanocrystals (NCs) per bacteriorhodopsin contributing to energy transfer can be determined from the inflection points of sigmoidal curves. This number was found to be highly dependent not only on the spectral overlap between $\mathrm{NC}$ emission and bR absorption bands, but also on nanocrystal surface charge. These results demonstrate the potential of how inorganic nanoscale materials can be employed to improve the generic molecular functions of biomolecules. The observed interactions between CdTe nanocrystals and bacteriorhodopsin can provide the basis for the development of novel functional materials with unique photonic properties and applications in areas such as all-optical switching, photovoltaics and data storage.
\end{abstract}

Keywords: quantum dots, semiconductor nanocrystals, bacteriorhodopsin, purple membranes, energy transfer, FRET, luminescence quenching, hybrid materials

\section{INTRODUCTION}

Although we rarely realise it, energy conversion and transfer processes play a massive role in life. Our bodies convert chemical energy to thermal and mechanical energy, whereas green plants convert solar energy to chemical energy via photosynthesis, thus producing new molecules and supramolecular structures. Another interesting example is bacteria Halobacterium salinarum, found in salted waters, which are able to survive extreme $\mathrm{pH}$ and ionic strength values, high pressures and a wide range of temperature variations ${ }^{1}$. Under such extreme conditions, the concentration of dissolved oxygen in such waters is often very low, and when it falls below that which is needed for respirative phosphorylation, the bacteria light-adapts the photochromic protein bacteriorhodopsin (bR) and switches to photosynthesis as a means of energy production ${ }^{2}$.

Patches of purple membranes (PMs) of several hundreds of nanometers in diameter and $5 \mathrm{~nm}$ in thickness can be extracted from bacteria ${ }^{3}$. In membranes, the bacteriorhodopsin is in the form of trimers, which are stabilised by 12 to 14 molecules of lipids and are arranged into a two-dimensional crystalline array (Figure 1c). This packing protects the protein from outside influences, with the result of an extraordinary thermal, chemical and photochemical stability of this protein $^{3}$. The chromophore element is a retinal (vitamin A aldehyde), which is bound to Lys-216 amino acid by a protonated Schiff base. The rest of the protein provides the proton conduction pathway and, along with the seven transmembrane $\alpha$-helices, protects the retinal from an attack by reactive chemical species, e.g. singlet oxygen and freeradicals $^{2}$. Upon absorption of light, the bacteriorhodopsin transports a proton from the intracellular to the extracellular side of the membrane, creating an electrochemical gradient across the membrane. A membrane bound ATPase, driven by this gradient, converts ADP to ATP which, in turn, is used for energising the cellular molecular processes ${ }^{2,3}$. During the

Photonic Materials, Devices, and Applications III, edited by Ali Serpengüzel, Gonçal Badenes, Giancarlo C. Righini, Proc. of SPIE Vol. 7366, 736620 - (c) 2009 SPIE · CCC code: 0277-786X/09/\$18 · doi: 10.1117/12.821731 
transport of a proton, bacteriorhodopsin goes trough a cyclic series of changes in molecular conformations - the intermediate states, all of which have distinctively different absorption spectra (Figures 1a and 1b).

Bacteriorhodopsin has received considerable attention based on its excellent photoelectric, photochromic or charge transfer properties ${ }^{3}$. Extensive research has been carried out in the use of this protein for the development of ultrafast light detectors, artificial retinas, motion detectors, 3-D and holographic information storage, associative memories, spatial light modulators, pattern recognition and optical filtering to name but a few.

While the basic mechanisms of the energy transfer and energy conversion phenomena described above have been thoroughly investigated, an appearance of the interfaces between the biological and nanosized non-organic moieties may produce very interesting effects. So, the multidisciplinary biological studies involving new technologies, such as nanotechnology, should certainly lead to breakthrough results in areas such as energy harvesting, transfer, conversion, storage and manipulation. Here, we report the first results of the engineering of a novel nano-bio hybrid material with advanced photonic properties, based on the unique properties of colloidal fluorescent quantum dots (Figure 1d) and the photochromic properties of bR membrane protein, for all-optical switching, photovoltaics and data storage applications.

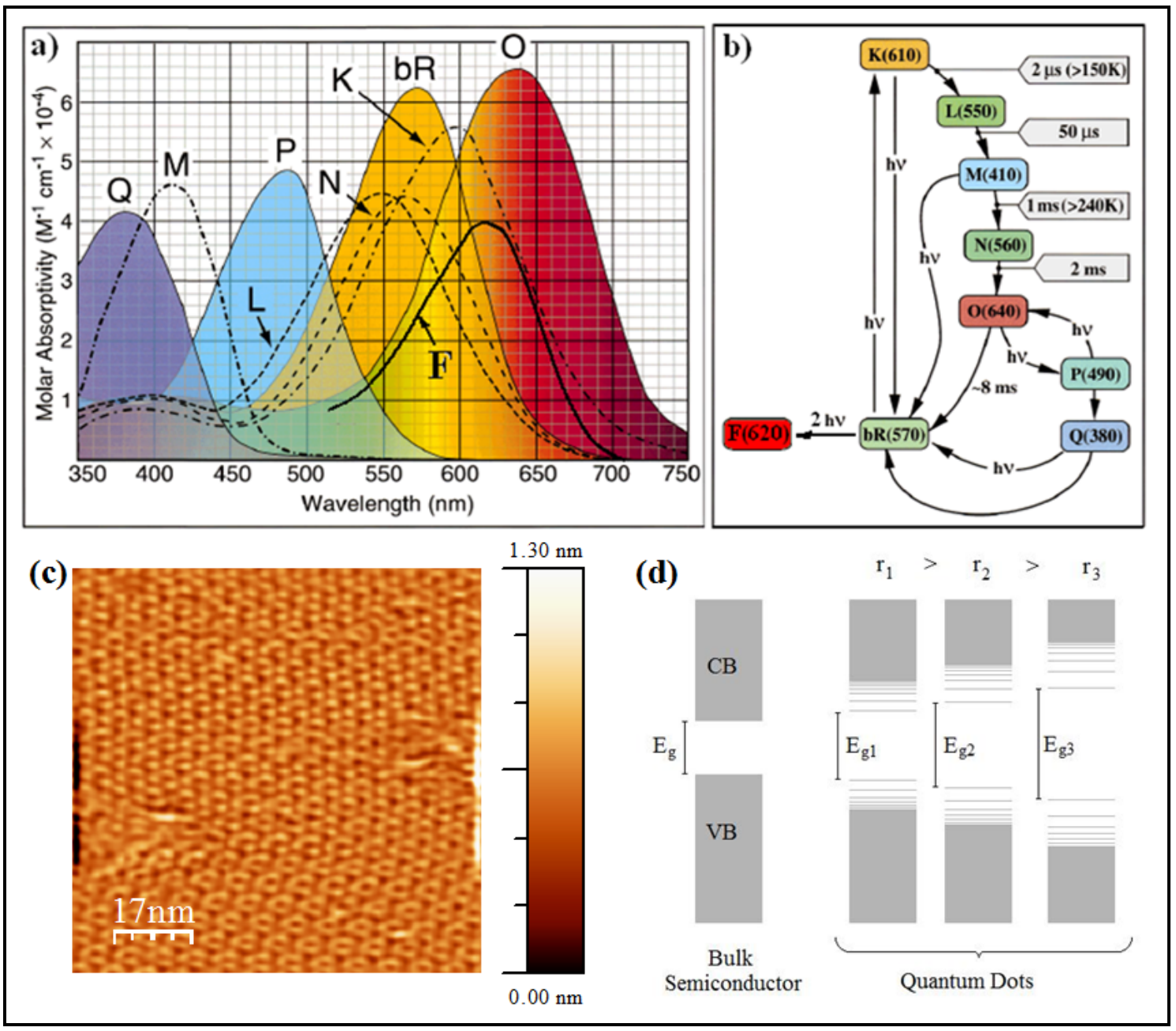

Fig. 1. (a) Ambient temperature absorption spectra of bacteriorhodopsin intermediate states and (b) diagram of bacteriorhodopsin photocycle, including lifetimes of excited intermediate states. Adapted from Birge et al. 1999. (c) An AFM image of a purple membrane showing a hexagonal array of bacteriorhodopsin trimers with unit dimensions of 6.2 $\mathrm{nm}$. (d) Schematic representation of the size-dependency of nanocrystal's band gap - as the size of the nanocrystal is decreased $\left(r_{1}>r_{2}>r_{3}\right)$, the band gap increases $\left(E_{g 1}<E_{g 2}<E_{g 3}\right)$. (for color image see electronic version) 


\section{MATERIALS AND METHODS}

\subsection{Materials and experimental description}

Wild-type bacteriorhodopsin (bR) powder was purchased from MIB GmbH. Thioglycolic acid (TGA)-stabilized CdTe NCs were synthesized and purified by previously reported methods ${ }^{4}$. Doubly purified deionized water from an $18 \mathrm{M} \Omega$ Millipore system was used for all dilutions.

Bacteriorhodopsin powder was dissolved in water to give a solution with absorbance of $\sim 0.2$ at $568 \mathrm{~nm}$. NCs were diluted with water to obtain clear solutions with absorbance of $\sim 0.15$ at the first excitonic peak. Both bR and QD stock solutions were sonicated for $10 \mathrm{sec}$ and 1 min respectively prior to measurements. The quantity of bR per QD was gradually increased by adding small volumes of bR stock solution to $1 \mathrm{~mL}$ of QD solution. After every addition, absorption, photoluminescence and lifetime measurements were performed. For the study of the $\mathrm{pH}$ response of the system, freshly prepared $1 \mathrm{M} \mathrm{NaOH}$ was used to raise the $\mathrm{pH}$ of solutions.

\subsection{Experimental methods and data treatment}

Absorption spectra were recorded on a Varian Cary50Conc UV-visible spectrophotometer. Steady-state photoluminescence measurements were carried out using a Varian CaryEclipse Fluorescence Spectrophotometer $\left(\lambda_{e x}=\right.$ $400 \mathrm{~nm})$.

PL decays were measured using time-correlated single photon counting utilizing a PicoQuant MicroTime200 set-up. Measurements were performed in ambient conditions, at room temperature, on solutions diluted to yield reasonable signal intensity. Samples were excited by a $480 \mathrm{~nm}$ picosecond laser pulse (PicoQuant LDH-480 laser head controlled by PDL-800B driver). The overall resolution of the set-up was $\sim 150 \mathrm{ps}$. The measured PL decays were reconvoluted using non-linear least-squares analysis. This was done with SymPhoTime software (PicoQuant) using an equation of the form

$$
I(t) \propto \sum_{i} \alpha_{i} \exp \left(-t / \tau_{i}\right)
$$

where $\tau_{i}$ are the PL decay lifetimes and $\alpha_{i}$ are the corresponding pre-exponential factors, taking into account the normalization of the initial point in the decay to unity. Weighted residuals and $\chi^{2}$ values were used to judge the quality of the fit. A fit with $\chi^{2}$ value of less than 1.1 was considered to be good. The $\tau_{i}$ and $\alpha_{i}$ values obtained from the fit were then used to calculate the average lifetime $\tau_{a v}$ using:

$$
\tau_{a v}=\frac{\sum \alpha_{i} \tau_{i}^{2}}{\sum \alpha_{i} \tau_{i}}
$$

\section{RESULTS AND DISCUSSIONS}

\subsection{Absorption studies}

Nanocrystals of three different sizes were used, emitting at $545 \mathrm{~nm}$ (green), $597 \mathrm{~nm}$ (orange) and $650 \mathrm{~nm}$ (red) respectively. These were chosen to provide varying degrees of overlap between the NC emission and bR absorption band (Figure 2a). Figures 1a to 1c show changes in the absorption spectra of the three NC solutions, as the amount of bR in them was increased. Absorption increased at wavelengths near $570 \mathrm{~nm}$ corresponding to an increase of bacteriorhodopsin concentration. Elsewhere, there was a decrease in absorption values due to a decrease in NC concentration. For all three NC solutions, a slight blue-shift of the peak (indicated by arrows in Figure 2) was observed. Overall, each spectrum of a NC-bR solution was a superposition of the absorption spectra of each component, indicating that no major chemical changes occur upon mixing of these two solutions 5 . 


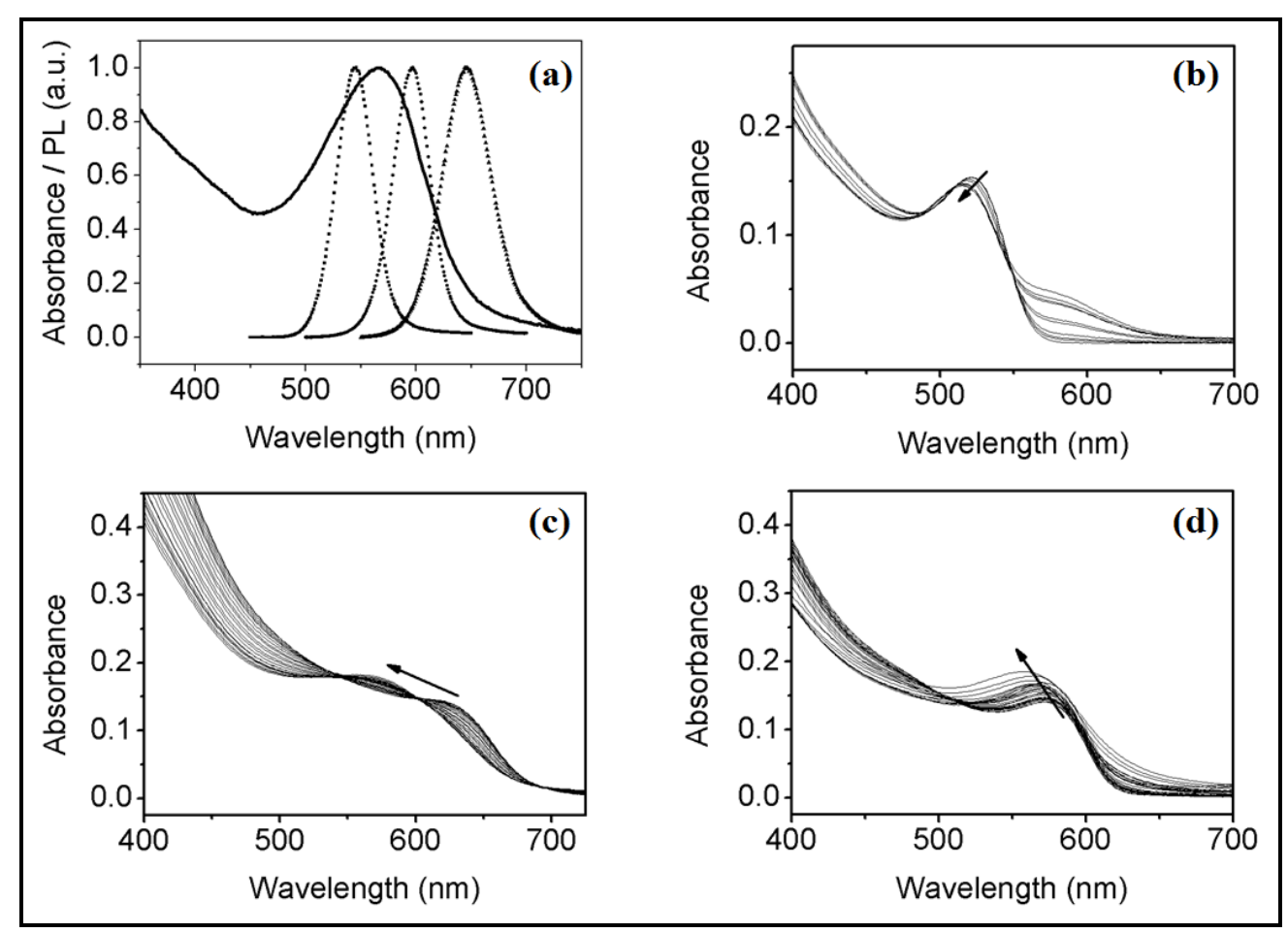

Fig. 2. (a) Emission spectra of $545 \mathrm{~nm}$ (green curver), $597 \mathrm{~nm}$ (orange) and $650 \mathrm{~nm}$ (red) nanocrystal samples. The purple curve is the bacteriorhodopsin absorption spectrum. Changes in absorption spectra of (b) green, (c) orange and (d) red QD solutions at increasing bacteriorhodopsin concentrations: the final MB:QD molar ratios were 0.33, 1.37 and 1.21 for green, orange and red QDs respectively. The arrows indicate the apparent blue-shifts in QD peaks.

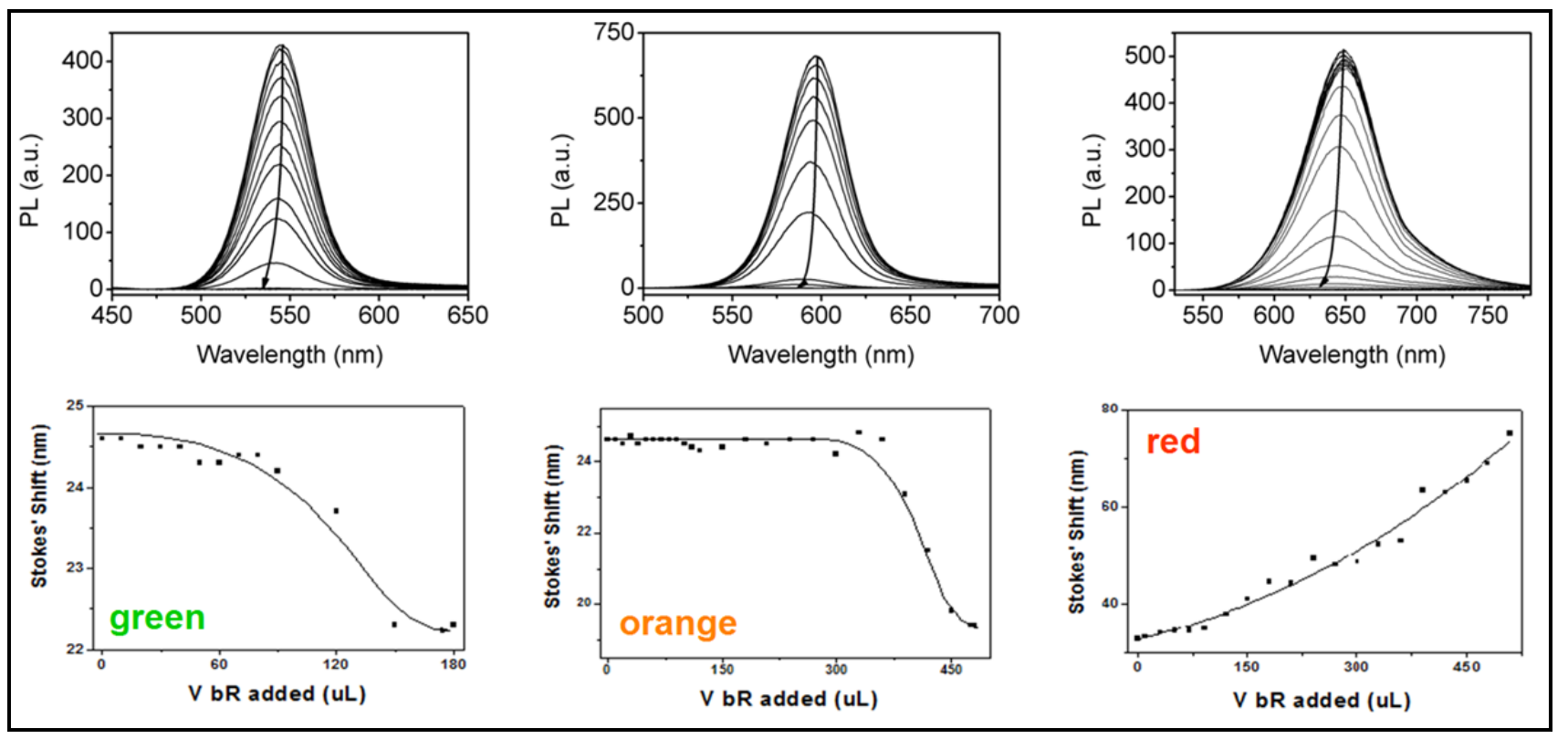

Fig. 3. Changes in photoluminescence spectra (top line) and corresponding stokes' shifts (bottom line) at increasing bacteriorhodopsin concentrations for green (first column), orange (second column) and red (third column) nanocrystals' samples. 


\subsection{Photoluminescence measurements and luminescence quenching mechanism}

Changes in luminescence were monitored by recording the photoluminescence spectra of solutions (Figure 3, top line). As the amount of $\mathrm{bR}$ was gradually increased, quenching of NC luminescence was observed. A blue shift in the PL peak was observed for all three samples, which seemed to be in accordance with shifts seen in absorption spectra. Stokes' shifts (Figure 3, bottom line), however, suggest that this is not the case. For green and orange NCs, the trend in Stokes' shifts was approximately sigmoidal, with a decrease in shifts with increasing protein concentration. A slightly concanve dependence was observed for the red NCs with an increasing Stokes' shift at increasing bR concentration. This can be attributed to the large difference in the absorbance maxima of the red NCs and bR, resulting in an enormous blue shift $(\sim 100 \mathrm{~nm})$ of the absorbance spectra of NCs at high bR concentrations. This shift by far outweighs the blue shift of the NC emission peak, resulting in the near-linear dependence of Stokes' shift for this NC sample. Overall, a non-constant Stokes' shift suggests that the blue shifts in emission spectra are not entirely due to blue shifts in absorbance spectra. This fact is the first indication that quenching of NCs' luminescence occurs through energy transfer between the CdTe nanocrystals and bacteriorhodopsin 6 .

In conjunction with photoluminescence studies, fluorescence lifetime measurements were also performed (Figure 4). For all three samples, a decrease in lifetimes was observed - this result is concurrent with the quenching via fluorescence resonance energy transfer (FRET) mechanism ${ }^{6}$.

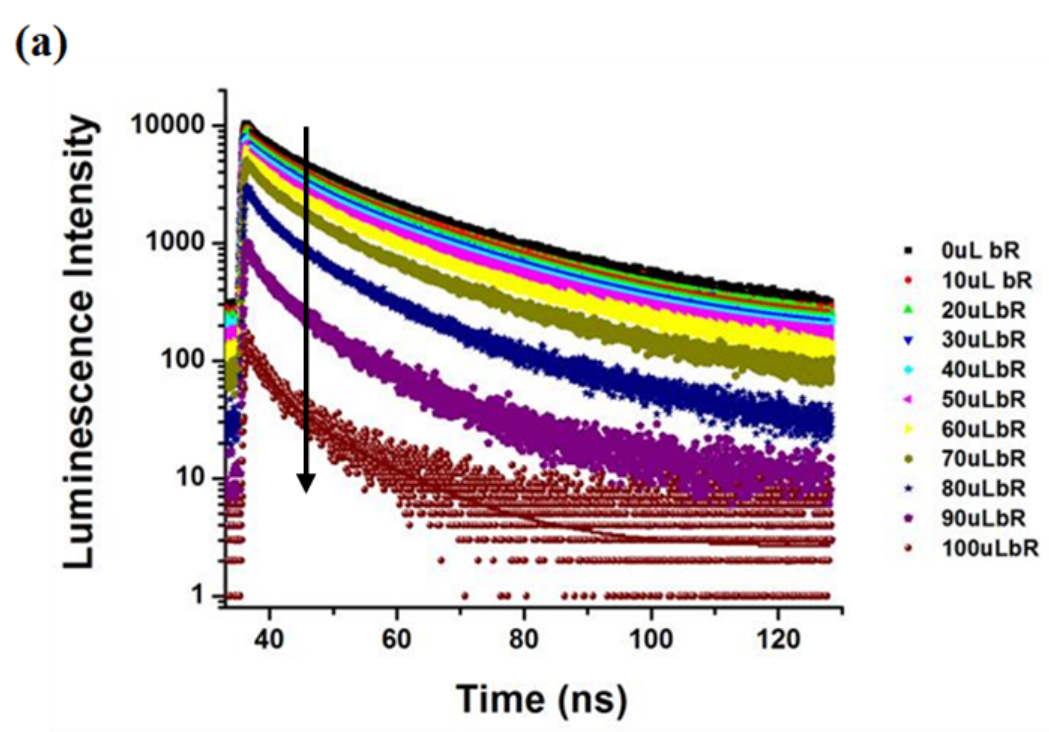

(b)

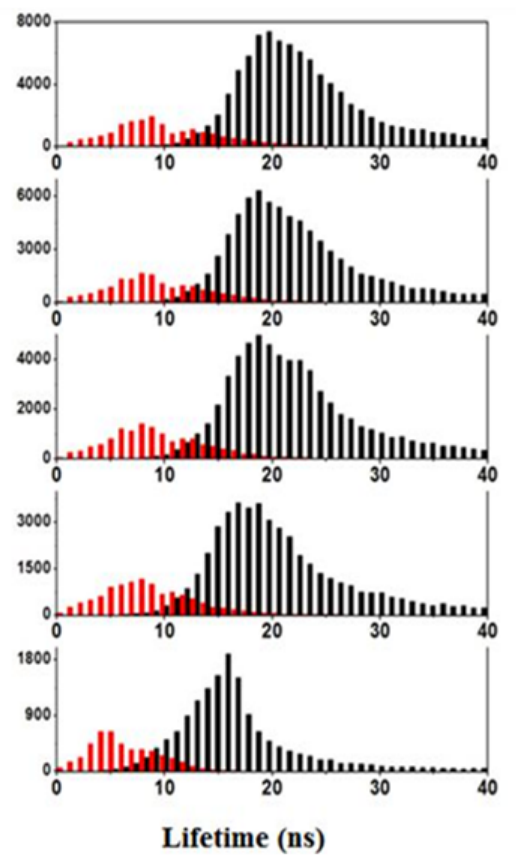

Fig.4. Time-resolved luminescence decay curves (above) and histograms of the two lifetimes obtained from the fitting procedure (left) at increasing bacteriorhodopsin concentrations. (for color image see electronic version)

\subsection{Nanocrystals-bacteriorhodopsin association}

The integrated area under the PL peak as a function of amount of bR in solution was found to strongly resemble a typical chemical titration curve. This resulted in good sigmoidal fits to the integrated PL data for all three samples, with $R^{2}$ values very close to unity (Figure 5a). This suggests that nanocrystals bind to the protein; their luminescence is then quenched through energy transfer. This is supported by non-linear intensity and lifetime Stern-Volmer plots for all three $\mathrm{NC}$ samples (Figure 5b), as well as the non-constant Stokes' shifts ${ }^{7}$. Most probably, this binding is of a purely electrostatic nature between the negatively charged NCs and the positive side of the purple membrane. 
To further test this conclusion, the luminescence intensity of $50 \%$ quenched green $\mathrm{NC}$ solution was measured while increasing the $\mathrm{pH}$ (Figure 6). A control experiment involved repetition of all measurements for an unquenched $\mathrm{NC}$ solution. Figure 6 and its inset show the final result of these measurements. The trends shown by the two solutions were found to be the opposite of each other. There was an initial increase in luminescence of the unquenched NC solution up to a $\mathrm{pH}$ of about 11 . This is to be expected since TGA-capped CdTe NCs were synthesized at this $\mathrm{pH}^{4}$. Partial deprotonation of $-\mathrm{COOH}$ groups of TGA stabiliser makes the NCs slightly more soluble, resulting in an increased luminescence intensity. At very high $\mathrm{pHs}(\geq 13)$, NCs become chemically unstable and the PL intensity decreases. For the $50 \%$ quenched NC solution, an initial decrease in luminescence intensity was observed (up to $\mathrm{pH}$ of $\sim 10$ ). This result suggests that some association between NCs and bR does indeed occur: as the $\mathrm{pH}$ of solution is increased, the NCs become slightly more charged and soluble and the binding between NCs and membranes is facilitated. Therefore, the decrease in PL intensity can be attributed to both the larger amount of NCs available to act as donors and/or tighter binding between the NCs and the membranes. At pHs greater than 10, the bR absorption band blue shifts (due to chemical changes) and falls off in intensity, resulting in a lesser overlap between NC emission and bR absorption bands. The bR ability to act as acceptor is thereby lessened and so NC luminescence intensity increases.

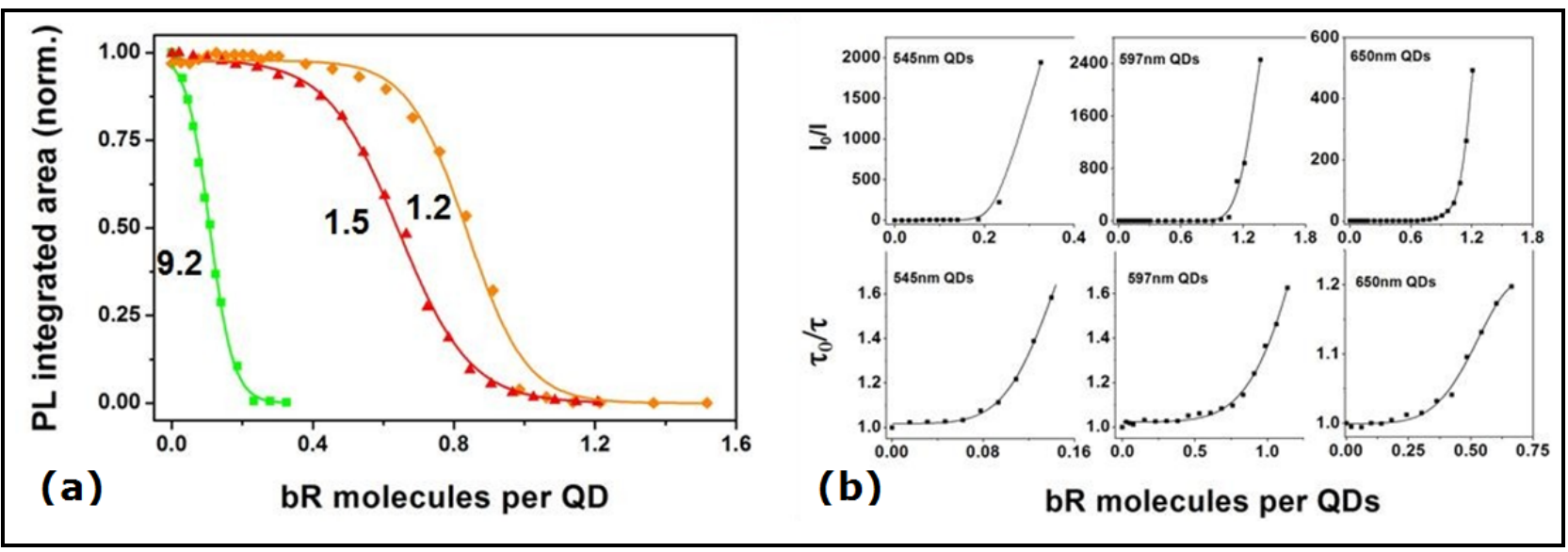

Fig. 5. (a) Integrated photoluminescence as a function of bacteriorhodopsin to quantum dot ratio quenching curves. The numbers indicated the number of nanocrystals that bind to the protein, that were obtained from sigmoidal fits to the data. (b) Intensity (top line) and lifetime (bottom line) Stern-Volmer plots. None of these curves are linear, suggesting that some association between nanocrystals and bacteriorhodopsin occurs.

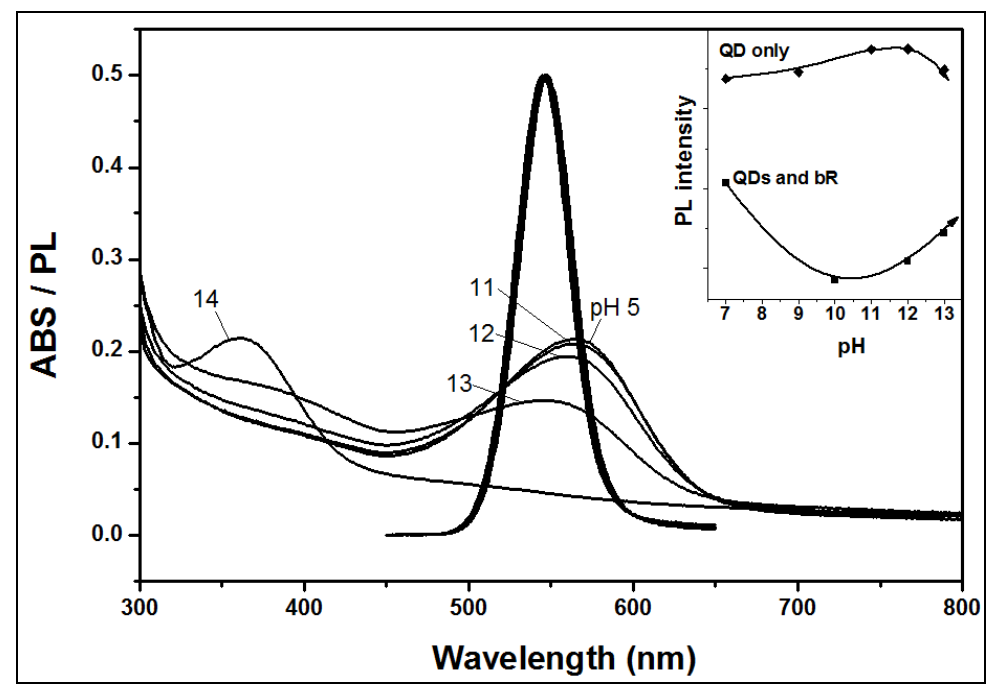

Fig. 6. Changes in absorption spectra and photoluminescence intensity values (inset) for $50 \%$ quenched NC solution at increasing $\mathrm{pHs}$. 


\subsection{Effect of nanocrystals' surface charge}

Based on the above reasoning, the sigmoidal plots can be considered to be an optical equivalent of chemical titration curves. The inflection points of the fits to this data then give the average number of NCs interacting with each protein (Figure 5a). This number $\left(N_{\text {int }}\right)$ was found to depend on the position of the NC emission band or, equivalently, on the size of the NCs. $N_{\text {int }}$ was larger for green NCs, at 9.2 NCs per bacteriorhodopsin. Orange and red NCs had 1.2 and 1.5 NCs per bR respectively (i.e. the number of NCs contributing to energy transfer was larger for red NCs than for orange NCs). However, if it is assumed that number of particles interacting with the protein only depends on the association constant between the bR and NC, which in turn directly relates to NC size (mass), the following trend is to be expected: $N_{\text {int }}$ (green) $>N_{\text {int }}$ (orange) $>N_{\text {int }}($ red $)$. This indicates that other factors, such as NC surface charge, may also play a role in the self-assembly of NCs and bR.

To test this, a control experiment was performed with a NC sample emitting close to $650 \mathrm{~nm}$ NCs above (i.e. at $645 \mathrm{~nm}$ ), but having a higher surface charge. The resulting quenching curve was compared to that for red NCs above (Figure 7). The luminescence of the $645 \mathrm{~nm}$ NCs (zeta potential of $-41 \pm 9 \mathrm{mV}$ ), was found to be quenched far quicker than that of the lesser charged $650 \mathrm{~nm} \mathrm{NCs}(-32 \pm 16 \mathrm{mV})$ with a corresponding increase in the number of NCs binding to bR from 1.5 for $650 \mathrm{~nm}$ NCs to 17.9 for $645 \mathrm{~nm}$ NCs (Figure 7). This explains the trend seen earlier for the orange and red NCs, since zeta potential measurements showed that the former NC sample had a slightly higher surface charge. Bearing in mind that the surface charge of NCs originates from the stabilizing molecules (ligands) on their surface, it is reasonable to assume that $\mathrm{NC}$ surface charge directly relates to the number of these ligand molecules per nanocrystal. But it is likely that this number will affect the thickness of ligand shell, and thus also the bR-NC separation distance. Thus, the effect we observed for the differently charged nanocrystals is due to the interplay between two effects: increased number of ligands per $\mathrm{NC}$ results in 1) the increased electrostatic interaction between the NCs and the protein, which aims to decrease the NC-bR separation distance, thereby increasing FRET efficiency between them; 2) the increase in the NC-bR separation due to increased ligand shell thickness, which has the opposite effect.

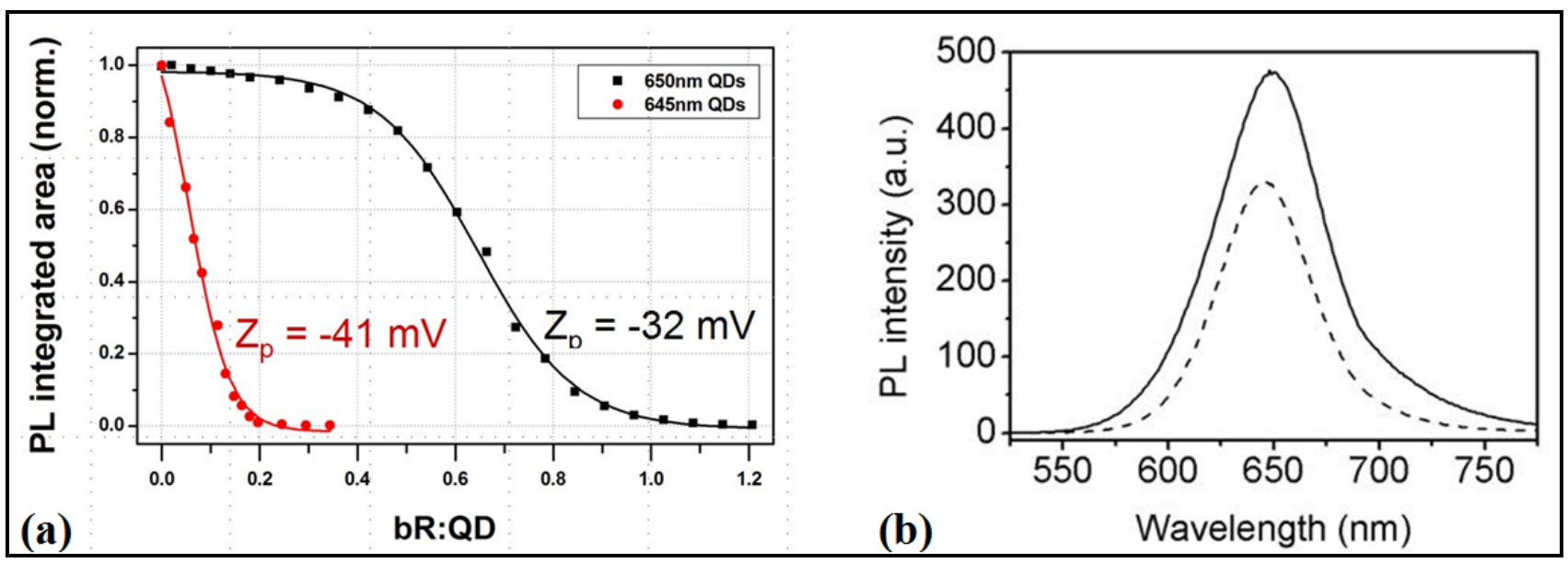

Fig. 7. (a) Integrated photoluminescence quenching curves for two NC samples emitting in the red (650 nm and $645 \mathrm{~nm})$ and their zeta potentials. (b) The emission spectra of $650 \mathrm{~nm}$ QDs sample (solid curve) and $645 \mathrm{~nm}$ QDs sample (dashed curve).

\section{CONCLUSIONS}

The potential impact of nanoscience on energy transfer processes in biomolecules was investigated on the example of the complex between fluorescent semiconductor nanocrystals and photochromic membrane protein bacteriorhodopsin. The interactions between colloidal CdTe QDs and bR protein were studied by a variety of spectroscopic techniques. Addition of bR to colloidal CdTe NCs was found to quench QDs' fluorescence. Decreasing emission lifetimes and non-constant Stokes' shifts at increasing protein concentrations suggest that this quenching occurs via Förster resonance energy transfer ${ }^{6}$. On the other hand, concave Stern-Volmer plots and sigmoidal PL quenching curves imply that the self- 
assembling of NCs and bR exists ${ }^{7}$, and the number of nanocrystals per bacteriorhodopsin contributing to energy transfer can be determined from the inflection points of sigmoidal curves. This number was found to be highly dependent not only on the spectral overlap between NC emission and bR absorption bands, but also on nanocrystals' surface charge.

The photochromic properties of bR provide the way to control the energy transfer between this protein and semiconductor QDs, paving the way for the development of all-optical switching, photovoltaic and light-harvesting devices based on this unique hybrid material.

\section{ACKNOWLEDGEMENTS}

This project was partly funded by the Embark Postgraduate Research Scholarship Scheme of the Irish Research Council for Science, Engineering and Technology (IRCSET). IN acknowledges support of the French National Research Agency (ANR). We would also like to thank Vladimir Lesnyak and Dr. Nikolai Gaponik for their academic assistance.

\section{REFERENCES}

[1] Hampp, N., "Bacteriorhodopsin as a Photochromic Retinal Protein for Optical Memories", Chem. Rev. 100(5), 1755 (2000)

[2] Birge, R. R., Gillespie, N. B., Izaguirre, E. W., Kusnetzow, A., Lawrence, A. F., Singh, D., Song, Q. W., Stuart, J. A., Seetharaman, S. and Wise, K. J., "Biomolecular electronics: Protein-based Associative Processors and Volumetric Memories”, J. Phys. Chem. B 103(49), 10746 (1999)

[3] Niemeyer, C. and Mirkin, C., [Nanobiothechnology: Concepts, Applications and Pespectives], Wiley, 146 (2004)

[4] Rogach, A. L., Katsikas, L., Kornowski, A., Dangsheng, S., Eychmuller A. and Weller H., "Synthesis and characterization of thiol-stabilized CdTe nanocrystals", Ber. Bunsen-Ges. Phys. Chem. 100(11), 1772-8 (1996)

[5] Bernard, M.-C., Hugot-Le Goff, A., Zeng, W., "Study by optical multichannel analysis, UV-vis-near-IR spectroscopy and Raman spectroscopy of the degradation mechanisms in a polyaniline/SnO2 electrochromic anode", Proc. SPIE 2528, 181-189 (1995)

[6] Clapp, A. R., Medintz, I. L. and Mattoussi, H., "Förster Resonance Energy Transfer Investigations Using QuantumDot Fluorophores", Chem.Phys.Chem. 7(1), 47 (2006)

[7] Lakowicz, J. R., [Principles of Fluorescence Spectroscopy], 2nd Edition, Springer, 368 (1999) 